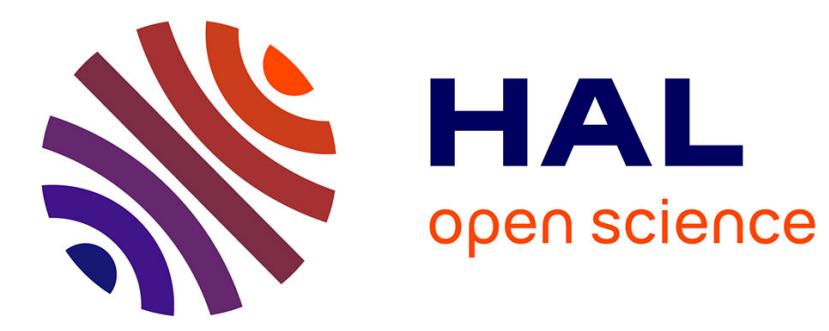

\title{
Réalisation d'un microscope ionique à champ
}

\author{
A. Menand, C. Martin, J. Gallot
}

\section{To cite this version:}

A. Menand, C. Martin, J. Gallot. Réalisation d'un microscope ionique à champ. Revue de Physique Appliquée, 1972, 7 (3), pp.177-180. 10.1051/rphysap:0197200703017700 . jpa-00243616

\section{HAL Id: jpa-00243616 https://hal.science/jpa-00243616}

Submitted on 1 Jan 1972

HAL is a multi-disciplinary open access archive for the deposit and dissemination of scientific research documents, whether they are published or not. The documents may come from teaching and research institutions in France or abroad, or from public or private research centers.
L'archive ouverte pluridisciplinaire HAL, est destinée au dépôt et à la diffusion de documents scientifiques de niveau recherche, publiés ou non, émanant des établissements d'enseignement et de recherche français ou étrangers, des laboratoires publics ou privés. 
Classification

Physics Abstracts

06.20

\title{
RÉALISATION D'UN MICROSCOPE IONIQUE A CHAMP
}

\author{
A. MENAND, C. MARTIN et J. GALLOT \\ Laboratoire de Microscopie Ionique, Faculté des Sciences de Rouen, 76-Mont-Saint-Aignan
}

(Reçu le 31 janvier 1972)

\begin{abstract}
Résumé. - Un microscope ionique à champ fonctionnant par pompage ionique sous ultravide a été réalisé. La température de l'échantillon peut atteindre $21^{\circ} \mathrm{K}$ et l'image est obtenue par conversion des ions en électrons puis multiplication de ceux-ci par une galette de microcanaux.

Abstract. - An ultra high vacuum field ion microscope employing an ionic pump has been designed. The sample temperature can be of the order of $21^{\circ} \mathrm{K}$ and the image is obtained by conversion of gas ions into electrons in a channel plate.
\end{abstract}

Un microscope à ionisation par effet de champ est constitué (Fig. 1) d'une enceinte dans laquelle est fait un vide élevé. L'échantillon à étudier est une

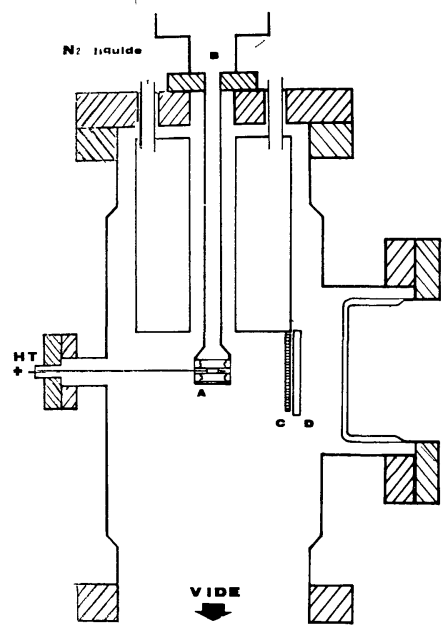

FIG. 1.

pointe très fine dont l'extrémité peut être assimilée à une portion de sphère de rayon compris entre 100 et $1000 \AA$. Cette pointe, maintenue à très basse température, est portée à un potentiel positif élevé par rapport à un écran placé en face. On introduit dans l'enceinte un gaz appelé " gaz image » (hélium, hydrogène, néon, argon ou mélange de ces gaz) à une pression de quelques milli-torr, les atomes de ce gaz sont ionisés sous l'action du champ très élevé (quelques volts par Å) qui règne au voisinage de la pointe et, accélérés par le champ électrique, vont ensuite frapper l'écran.

Le champ $E_{\mathrm{I}}$ nécessaire pour ioniser le gaz image dépend essentiellement de l'énergie d'ionisation de l'atome de gaz. Les champs $E_{1}$ caractéristiques des différents gaz image utilisés sont : 4,5 $\mathrm{V} / \AA ̊$ (hélium), $3,4 \mathrm{~V} / \AA ̊$ (néon), 1,9 $\mathrm{V} / \AA ̊$ (argon) [1].
La densité de courant ionique est la plus grande dans les régions de l'espace où le champ est le plus intense : c'est-à-dire au voisinage des protubérances, à l'échelle atomique, de la pointe. L'image formée sur l'écran peut être assimilée approximativement à la projection stéréographique de la surface de l'échantillon.

L'ordre de grandeur du grandissement de l'image est grossièrement donné par le rapport de la distance pointe-écran au rayon de courbure de la pointe, il est généralement de plusieurs millions. La structure, à l'échelle atomique, de la surface de l'échantillon, est alors observée avec un pouvoir de résolution de 2 à $3 \AA[2]-[3]$.

Pour une certaine valeur $E_{\mathrm{E}}$ du champ électrique il y a évaporation par effet de champ des atomes de la surface. Pour avoir une image ionique stable [4] il faut nécessairement que :

$$
E_{\mathrm{E}}>E_{\mathrm{I}} \text {. }
$$

Comme $E_{\mathrm{E}}$ dépend de la nature des atomes constituant la pointe, pour imager les métaux non réfractaires et respecter la condition précédente, il faut diminuer $E_{\mathrm{I}}$ et prendre des gaz images comme néon et argon.

Réalisation du microscope. - VIDE. - L'emploi de l'hélium élimine la contamination de l'échantillon par les impuretés présentes dans l'enceinte car cellesci sont ionisées pour un champ électrique inférieur à $4,5 \mathrm{~V} / \AA$ donc loin de la surface.

Avec le néon ou l'argon, la pureté de l'atmosphère de l'enceinte devient critique. Ceci conduit à l'emploi de l'ultra-vide et à la purification du gaz image. A cet effet, le microscope est équipé d'un groupe à ultravide constitué de trois systèmes de pompage : une pompe à palette SOGEV BL 15, deux pompes à sorption SOGEV S 40Z 110, une pompe ionique triode SOGEV $250 \mathrm{l} / \mathrm{s}$ couplée à un sublimateur de titane. 
L'ensemble ultra-vide ainsi que l'enceinte sont étuvables à $350^{\circ} \mathrm{C}$. Le vide est alors de quelques $10^{-10}$ torr. La pompe ionique a été surdimensionnée pour garder une vitesse de pompage raisonnable des gaz rares.

La pureté de l'atmosphère est d'autant plus critique que, par l'emploi des intensificateurs d'image, la pression de gaz image utilisée est de $10^{-5}$ à $10^{-6}$ torr au lieu de $10^{-3}$ torr sans intensificateur ce qui augmente le taux d'impureté provenant des parois du microscope.

Mise EN FROID DE L'ÉCHANTILlON. - La résolution du microscope est limitée par la vitesse tangentielle des ions du gaz image due à l'agitation thermique. Afin d'obtenir la résolution atomique, l'échantillon est refroidi à la température de l'hydrogène liquide à l'aide d'un microliquéfacteur Air Liquide - Philips RC 1000 étuvable. Le porte-échantillon doit être un bon conducteur thermique et un bon isolant électrique ; l'alumine a été choisie pour sa commodité d'emploi.

Haute tension. - La haute tension appliquée à la pointe est fournie par un générateur électrostatique SAMES D 30. Cet appareil délivre une tension positive réglable de façon continue de $1 \mathrm{kV}$ à $30 \mathrm{kV}$, de stabilité $<10^{-4}$.

Le générateur haute tension est relié à un générateur d'impulsions réalisé par la SAMES. Ceci permet de superposer à la tension continue une impulsion rectangulaire de hauteur variable entre 300 et $3000 \mathrm{~V}$, de largeur réglable de 5 à $50 \mu$ s et de temps de montée inférieur à $1 \mu \mathrm{s}$. Les impulsions peuvent être produites manuellement ou automatiquement, la fréquence de répétition étant alors réglable de 1 à 10 impulsions par seconde. Ce montage permet un contrôle fin de l'évaporation par effet de champ (quelques atomes $\mathrm{du}$ bord d'un plan atomique évaporés par pulse). De plus, la tension continue est mesurée à travers un pont diviseur de rapport 1 à $10^{4}$ à l'aide d'un voltmètre digital 4 décades SCHNEIDER VN 454 sur la sensibilité $3 \mathrm{~V}$.

Ceci permet en particulier de mesurer le potentiel pour lequel certains atomes de la pointe s'évaporent par effet de champ et de comparer les énergies de liaisons des atomes en surface suivant leur situation et leur nature.

INTENSIFICATEURS D'IMAGES. - Lorsqu'on veut passer de l'obtention d'une image ionique aux applications de la microscopie ionique, le facteur temps nécessaire pour obtenir des résultats valables devient prépondérant. En effet, une étude tridimensionnelle d'une pointe par évaporation couche par couche des atomes de la surface demande des millions de micrographies.

En utilisant l'hélium comme gaz image, les temps de pose sont de l'ordre de 10 minutes. Pour obtenir des images ioniques de métaux moins réfractaires
( $\mathrm{Cu}$ ou $\mathrm{Al}$ par exemple), il est nécessaire d'utiliser des gaz dont le potentiel d'ionisation est plus faible que celui de l'hélium. Le néon ou l'argon conviennent pour ce type d'étude mais l'efficience des ions argon ou néon sur le phosphore est très faible et ils détruisent très rapidement l'écran fluorescent. Il est donc indispensable de posséder, pour faire de la microscopie ionique, un intensificateur d'images.

Plusieurs solutions ont été utilisées : Amplificateur de brillance externe qui ne supprime pas complètement les inconvénients rencontrés dans l'utilisation de gaz comme le néon ou l'argon, puis la conversion des ions en électrons par une grille - les électrons produits sont focalisés sur l'écran par un champ magnétique et électrique. Mais, le progrès le plus important a été l'utilisation de galettes de microcanaux. Celles-ci sont constituées d'un réseau de multiplicateurs tubulaires.

Les performances et leurs applications ont été décrites dans deux volumes d'Acta Electronica [6], [7].

Pour diminuer les temps d'exposition et augmenter les possibilités du microscope, nous avons monté une galette de multiplicateurs tubulaires (Channel plate) BENDIX de deux pouces de diamètre. La galette est constituée par un réseau très serré de multiplicateurs tubulaires, chàque tube convertit d'abord les ions en électrons qui sont ensuite multipliés en cascade à l'intérieur du tube. En sortant de celui-ci, les électrons sont accélérés par un champ électrique coaxial au tube et frappent un écran très proche de la face de sortie de la galette.

Les détails de ce montage sont indiqués sur la figure 2. La face avant de la galette est mise à la masse

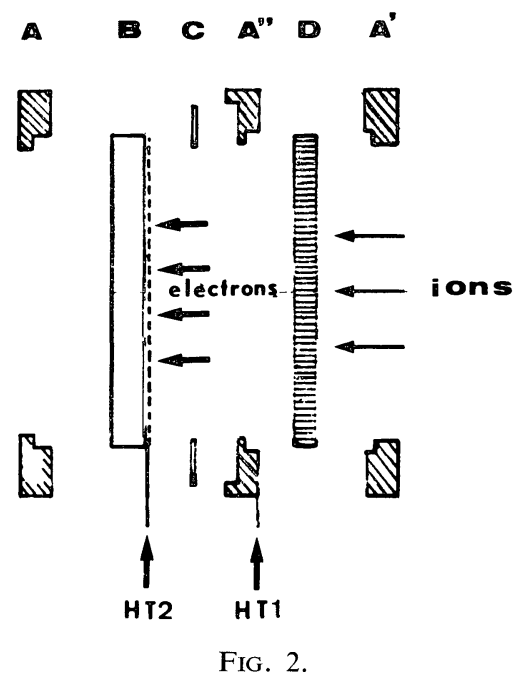

par la pièce en inox $\mathrm{A}^{\prime}, \mathrm{A}^{\prime \prime}$ maintient la galette en bonne position et étant relié à une haute tension $\mathrm{HT}_{1}$ porte la face arrière à un potentiel convenable. L'écran de verre $\mathrm{B}$ est positionné par la pièce $\mathrm{A}$, la face conductrice recouverte de poudre luminescente est reliée à la haute tension $\mathrm{HT}_{2}$ au moyen d'une feuille de tantale de 5/100 mm d'épaisseur. Elle est isolée de la haute 
tension $\mathrm{HT}_{1}$ par la rondelle de verre $\mathrm{C}$ de $5 / 10 \mathrm{~mm}$ d'épaisseur. La distance B-D (écran-galette) doit être aussi faible que possible pour éviter une dispersion des électrons qui nuirait à la résolution; d'autre part, la différence de potentiel entre $\mathrm{B}$ et $\mathrm{D}$ est importante et impose une valeur limite à $\mathrm{BD}$. Dans notre montage, cette distance est inférieure à $8 / 10 \mathrm{~mm}$ et pour une différence de potentiel de $5 \mathrm{kV}$ et une pression de $1 \times 10^{-4}$ torr, il n'y a pas de phénomène d'arc.

A une pression de "gaz image » de $2 \times 10^{-5} \mathrm{~mm}$ de $\mathrm{Hg}$ le gain en brillance est de l'ordre de $10^{4}$ avec $\mathrm{HT}_{1}$ et $\mathrm{HT}_{2}$ respectivement égales à $800 \mathrm{~V}$ et à $4000 \mathrm{~V}$.

La comparaison des figures' 3 et 4 montre le gain de temps de pose obtenu avec l'emploi de l'intensificateur, malgré une pression de gaz imageant 100 fois plus petite.

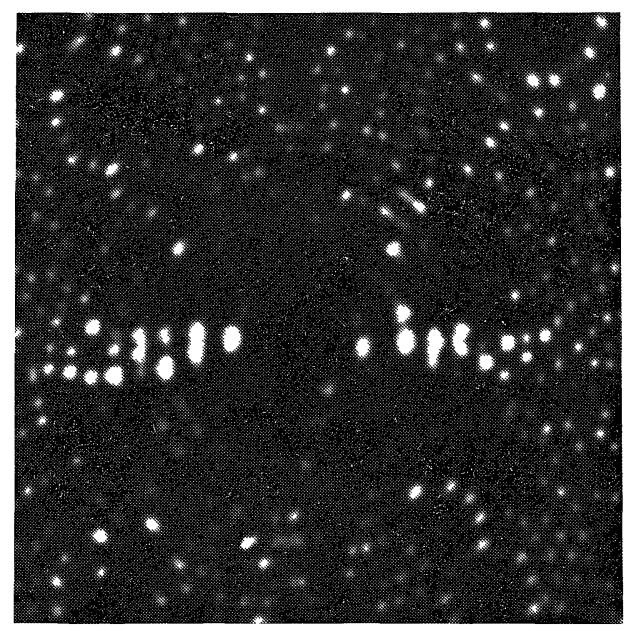

FIg. 3. - Tungstène à $21^{\circ} \mathrm{K}$. Pression d'Hélium $3 \times 10^{-3}$ torr. Photo prise sur film Kodak tri $\times \mathrm{f}: 2$, temps de pose 5 minutes.

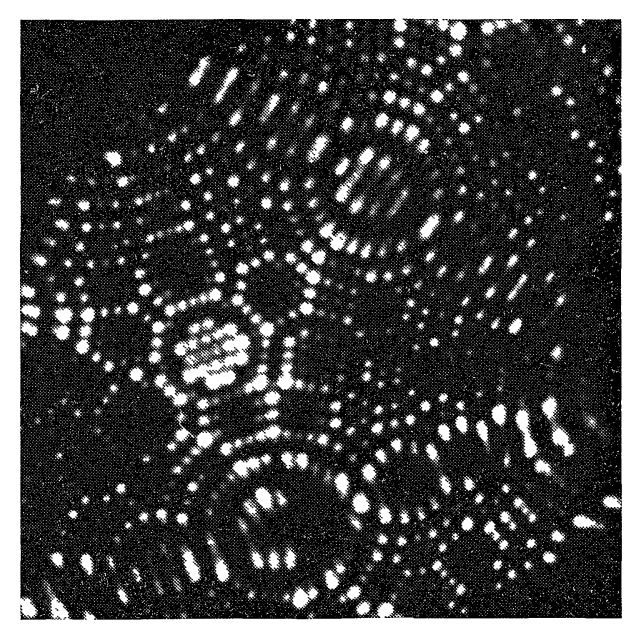

Fig. 4. - Platine à $21^{\circ} \mathrm{K}$. Pression d'hélium 1,5 × 10-5 torr. Photo prise sur film Kodak plus $\times f: 8$, temps de pose 1 seconde.

Le microscope ionique ainsi construit est utilisable pour différents types d'études. La température voisine de $21^{\circ} \mathrm{K}$ de l'échantillon augmente le pouvoir séparateur et la gamme de métaux et alliages qu'il est possible d'étudier. L'intensificateur d'images permet de faire des études statistiques dans un temps raisonnable par la possibilité d'enregistrement rapide des micrographies.

La propreté du vide obtenu et la mesure du potentiel de désorption des atomes conduisent aux études de surfaces.

Nous donnons maintenant deux exemples représentatifs des performances du microscope utilisant la résolution atomique.

Etude d'une solution solide [8]. - Cette étude a été faite sur platine or à $5 \%$ d'or qui répond aux deux conditions nécessaires pour interpréter les micrographies ioniques. D'abord l'image doit être régulière et présenter les symétries caractéristiques de l'alliage étudié. Ensuite les deux types d'atomes doivent pouvoir être distingués. Cette dernière condition correspond à l'influence de la nature de l'atome sur l'ionisation ou l'évaporation par effet de champ. Il y a dans certains cas, soit évaporation préférentielle d'un type d'atome, soit ionisation sélective audessus d'un type d'atome. S'il est difficile de prévoir théoriquement [9], quel contraste donnera un alliage donné. Il est possible de trouver une solution expérimentale à ce problème.

Dans le cas des alliages platine or, la concentration en atomes manquant sur certains plans atomiques est égale à la concentration en or [10]. En admettant que toutes les « lacunes » dans les plans (210) de la micrographie ionique de l'alliage platine or à $5 \%$ sont des atomes d'or (Fig. 5), il est possible de trouver les

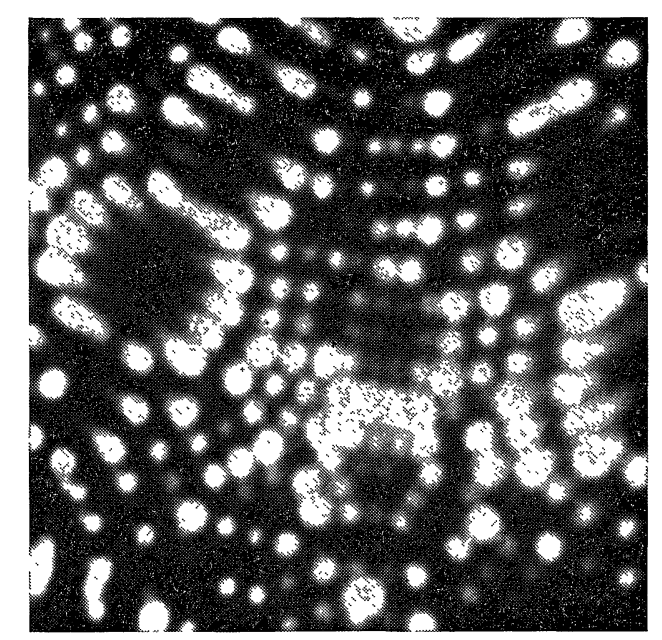

FIG. 5. - Plan (210) du platine montrant 3 atomes d'or. Gaz image $\mathrm{He} T=21^{\circ} \mathrm{K}$.

coordonnées des atomes d'or dans ce plan. Par évaporation de ce plan qui se fait très progressivement, on relie ces coordonnées au plan situé immédiatement sous la surface. Ceci permet d'avoir directement le réseau tridimensionnel des atomes d'or dans une matrice de platine et d'obtenir des informations sur l'ordre et la statistique des solutions solides. 


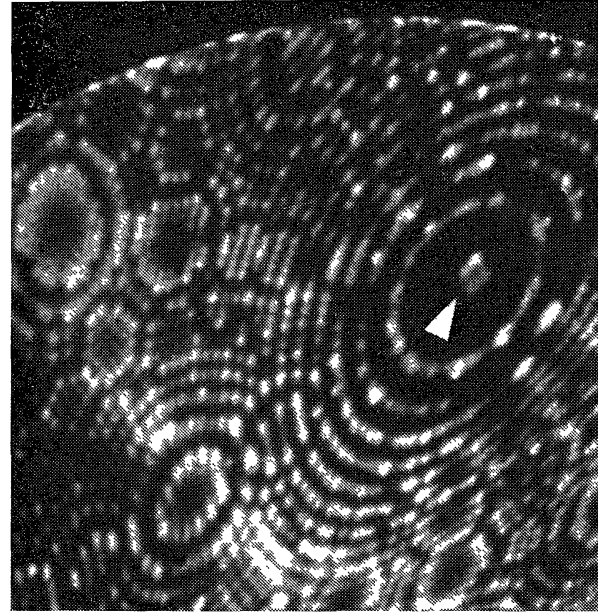

FIG. 6.

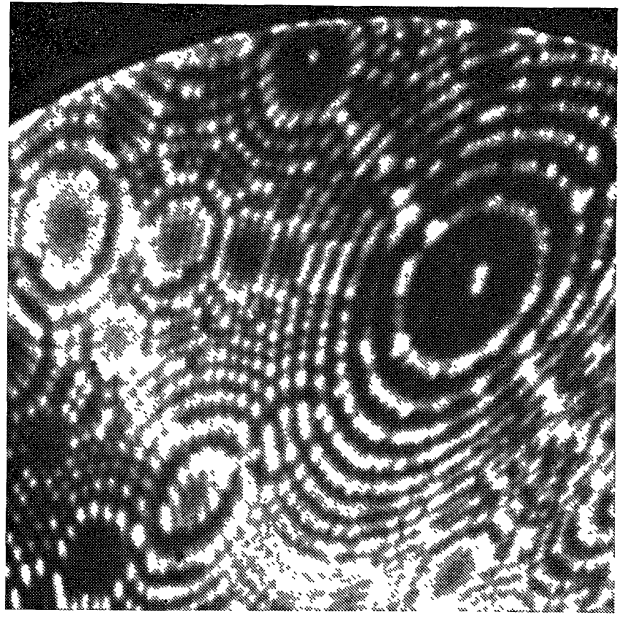

FIG. 7.

Iridium à $21^{\circ} \mathrm{K}$. Pression d'Hélium $2,5 \times 10^{-5}$ torr. Photo prise sur film ILFORD HP 4 F : 8 , temps de pose $1 / 2$ seconde.

Etude d'une surface. - La résolution du microscope ionique et la possibilité d'obtenir par évaporation par effet de champ une surface propre permettent d'envisager les applications dans le domaine de la physique des surfaces.

Nous avons utilisé le microscope pour étudier l'énergie de liaison d'atomes déposés sur la surface de la pointe [11]. En effet, le champ d'évaporation d'un atome donné dépend de son énergie de liaison. L'atome s'évapore par effet de champ sous la forme d'un ion $n$ fois chargé.

$$
E_{\mathrm{E}} \simeq \frac{1}{n^{3} e^{3}}\left(\Lambda+\sum_{i=1}^{n} I_{\mathrm{i}}-n \Phi\right)^{2}
$$

$\Lambda$ énergie de liaison 'de l'atome à la surface,

$I_{\mathrm{i}}$ énergie d'ionisation de l'atome,

$\Phi$ travail de sortie.

Pour faire cette mesure, il est nécessaire de contrôler l'évaporation et de mesurer le potentiel d'évaporation.

Le contrôle de l'évaporation est réalisé à l'aide $\mathrm{du}$ générateur d'impulsions.

Ces impulsions sont superposées à une tension continue délivrée par un générateur électrostatique et mesurée par le voltmètre digital.

Un évaporateur permet de déposer sur l'échantillon les atomes dont on veut étudier la désorption.
Il est constitué de deux filaments de métaux à étudier séparés par une feuille de tantale.

Les photos 6 et 7 montrent une séquence d'évaporation contrôlée d'une pointe d'iridium. Sur la première photo, il reste 4 atomes d'iridium (indiqués par la flèche) sur le plan 100, deux de ces quatre atomes partent à $12150 \mathrm{~V}$ et il reste les deux atomes visibles sur la photo $\mathrm{n}^{0} 7$; ceux-ci partent à $12000 \mathrm{~V}$.

Conclusion. - Les performances de ce microscope sont satisfaisantes. Cet" appareil est cependant limité pour l'utilisation des "gaz imageants 》 autres que l'hélium à un fonctionnement statique dû au mauvais comportement des pompes ioniques en présence de néon ou d'argon. Le fonctionnement en pression dynamique de gaz imageant est en effet préférable pour éviter de polluer trop rapidement l'atmosphère du microscope par les gaz désorbés par les parois. Enfin, sa mise en fonctionnement est assez longue après chaque changement d'échantillon.

Pour ces deux raisons, il est envisagé un pompage annexe par pompe turbomoléculaire et la construction d'un changeur d'échantillons.

Remerciements. - Nous remercions la DRME et la DGRST pour l'aide apportée, cette étude a été effectuée sous contrat.

\section{Bibliographie}

[1] Van Oostrom (A.), Philips Rev. Repts, 1970, 25, 85. [2] Mueller (E. W.), Tsong (T. T.), Field Ion microscopy principles and applications, Elsevier, 1969.

[3] Bowkett (K. M.), Smith (D. A.), Field Ion microscopy defect in crystalline solids, vol. 2, 1970.

[4] Gomer (R.), Swanson (L. W.), J. Chem. Phys., 1963, 38, 1613.

[5] Turner (P. J.), Cartwright (P.), Southon (M. J.), van Oostrom (A.), Manley (B. W.), J. Sci. Instr., 1969, 2, 731 .
[6] Acta Electronica, 1971, 14, $\mathrm{n}^{\circ} 1$.

[7] Acta Electronica, 1971, 14, no 2.

[8] Martin (C.), Gallot (J.), A paraître.

[9] Soutworth (H. N.), RAlPH (B.), J. of Microscopy, 1969, 90, 167.

[10] Dubroff (W.), Machlin (E. S.), Acta Met., 1968 , $16,1313$.

[11] Plummer (E. W.), Rhodin (T. N.), J. Chem. Phys., $1968,49,3479$. 